\title{
General cracked-hinge model for simulation of low-cycle damage in cemented beams on soil
}

Skar, Asmus; Poulsen, Peter Noe; Olesen, John Forbes

Published in:

Engineering Fracture Mechanics

Link to article, DOI:

10.1016/j.engfracmech.2017.01.016

Publication date:

2017

Document Version

Peer reviewed version

Link back to DTU Orbit

Citation (APA):

Skar, A., Poulsen, P. N., \& Olesen, J. F. (2017). General cracked-hinge model for simulation of low-cycle damage in cemented beams on soil. Engineering Fracture Mechanics, 175, 324-338.

https://doi.org/10.1016/j.engfracmech.2017.01.016

\section{General rights}

Copyright and moral rights for the publications made accessible in the public portal are retained by the authors and/or other copyright owners and it is a condition of accessing publications that users recognise and abide by the legal requirements associated with these rights.

- Users may download and print one copy of any publication from the public portal for the purpose of private study or research.

- You may not further distribute the material or use it for any profit-making activity or commercial gain

- You may freely distribute the URL identifying the publication in the public portal 


\title{
General cracked-hinge model for simulation of low-cycle damage in cemented beams on soil
}

\author{
Asmus Skar ${ }^{1, *}$ \\ COWI A/S, Parallelvej 2, 2800 Kgs. Lyngby, Denmark \\ Peter Noe Poulsen², John Forbes Olesen², \\ Technical University of Denmark, Brovej, Building 118, 2800 Kgs. Lyngby, Denmark
}

\begin{abstract}
The need for mechanistic constitutive models to evaluate the complex interaction between concrete crack propagation, geometry and soil foundation in concrete- and composite pavement systems has been recognised. Several models developed are either too complex or designed to solve relatively simple problems, e.g. limited to one type of load configuration or test setup. In order to develop a general and mechanistic modeling framework for non-linear analysis of low-cycle damage in cemented materials, this paper presents a cracked-hinge model aimed at the analysis of the bending fracture of the cemented material. The model is based on the fracture mechanics concepts of the fictitious crack model. The proposed hinge is described in a general and consistent format, allowing for any type of stress-crack opening relationship and unloading- reloading formulation. The functionality of the proposed hinge model is compared to numerical- and experimental results. The proposed hinge shows good performance and seems promising for the description of low-cycle fracture behavior in cemented materials.
\end{abstract}

Keywords: Fracture mechanics, cohesive crack, non-linear FEM, low-cyclic fatigue, cemented materials, pavement analysis

\footnotetext{
${ }^{*}$ Corresponding author

Email address: asch@cowi.dk (Asmus Skar)

${ }^{1}$ Industrial Researcher

${ }^{2}$ Associate Professor
} 


\section{Introduction}

Concrete- and composite pavement systems are subjected to cyclic loading from vehicles resulting in initiation of bending cracks in the quasi-brittle cemented material. Subsequently, these cracks propagate leading to failure of the pavement structure. The structural design of such pavements is primarily based on empirical formulas which convert the elastic response analysis into a measure of performance $[1,2,3]$, referred to as the Mechanistic-Empirical (M-E) method. However, such a method cannot account for significant factors influencing the response, e.g. describing the interaction between loads, material properties, geometry and soil foundation in a unified manner.

The limitations of the M-E method and the growth in computer capabilities have resulted in an increasing development of more rational models for pavement analysis during the past decades. That work began in the early 1990s, studying mainly asphalt concrete mixtures in flexible pavements $[4,5,6]$, reflective cracking in asphalt overlays [7] and permanent deformation of unbound materials $[8,9,10]$. These models are typically based on a mechanistic approach using appropriate numerical tools, e.g. the finite element (FE) method. This allows for geometry, inhomogeneities, anisotropy, and nonlinear material properties of all pavement layers to be considered.

Numerical analysis of crack propagation in concrete- and composite pavement systems have primarily carried out applying cohesive zone modeling $[11,12,13,14,15]$. Gaedicke and Roesler [16, 17] applied a cohesive zone model for studying fracture in beams resting on soil. The cohesive zone model stand out as particularly attractive for studying beams- and slabs on grade under monotonic loading, where the fracture plane can be anticipated a-priori, i.e. orthogonal cracks in the direction of maximum principal stress. Although the results published show good performance, relatively large finite element models are applied compared to the complexity of the pavement system studied. Moreover, some authors reported lack of convergence, instability problems and aborted simulations [16, 13, 14].

In order to create a simple and robust modeling framework for engineering application, this paper presents a non-linear hinge model based on the fracture mechanics concepts of the fictitious crack model by Hillerborg et al. [18]. The hinge model for modeling the crack propagation due to pure bending in a concrete beam without reinforcement was first presented by Ulfkjaer et al. [19]. This hinge was successfully applied in the modeling of pure con-

crete beams in three-point bending considering the development of only one 
crack. Olesen [20] expanded the hinge model by applying a bi-linear softening curve to allow for the incorporation of the effects of fibers on concrete fracture. Further, this modified hinge model allowed for the existence of a sectional normal force. This last feature is crucial for the ability of the hinge to model a number of situations such as the wedge splitting test [21] and the split cylinder test [22]. Olesen and Poulsen [23] implemented a numerical hinge for investigation of the fracture behaviour of reinforced concrete beams applying the effective stiffness concept, allowing for the formation of multiple cracks along the beam axis.

The basic idea of the hinge has also been used for studying cyclic fatigue of plain concrete beams $[24,25,26]$, as well as reinforced and fiberreinforced concrete beams [27, 28, 29, 30]. Maitra et al. [31] applied the influence method [32] in conjunction with the stress-degradation law proposed by Zhang et al. [25] to simulate fatigue crack length propagation in plain concrete pavements. However, these models suffer from another drawback: they are limited to one type of test or structural problem where the crack path is known a-priori.

This paper presents a simple multi-scale damage model for simulation of low-cyclic damage of cemented beam structures. At the lowest level, a fiber of cemented material including a crack is considered, and a stress-mean strain relationship is established. At the intermediate level, a hinge element, which is a finite part of the beam, consisting of fibers in layered strips of cemented material is considered, and a relationship between the generalised sectional forces and strains established. At the highest level, the hinge model is applied as a constitutive model in a non-linear beam element. Although the underlying description of the hinge is based on the formation of discrete cracks, the constitutive behavior of the hinge is smeared (smooth). This particular feature is practical and effective as no a-priori knowledge of the crack pattern is required. Moreover, the hinge formulation makes it straightforward to implement different types of stress-crack opening relationships and unloading- reloading schemes.

\section{The mechanics of the cracked-hinge model}

The basic assumption of the hinge model is the fact that the presence of a crack influences the overall stress and strain field of a structure only locally. The discontinuity created by the crack is expected to vanish outside a certain width. Under constant moment, e.g. between the loaded points in Figure 
1 (a), the beam sections at the midpoints between the cracks will, due to the periodicity of the cracks, remain plane during deformation of the beam. The width $s$ between two such sections embracing one crack defines a hinge element, as shown in Figure 1 (b). For the beam area outside the loaded points, the moment distribution is no longer constant. Such phenomena be handled with appropriate numerical tools, i.e., the finite element (FE) method, as exemplified for a single beam element in Figure 1 (b).

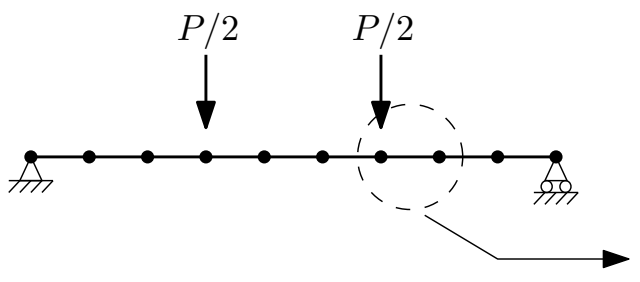

(a)

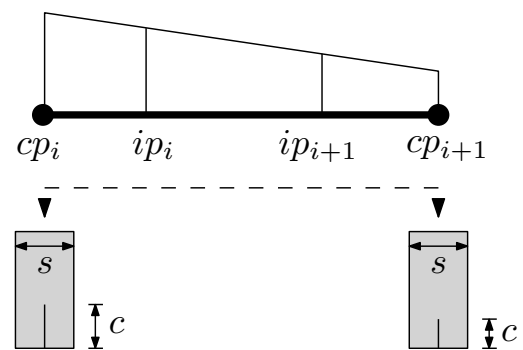

(b)

Figure 1: Sketch of hinge model implemented in simply supported beam under four point loading: (a) overview of beam structure, (b) underlying discrete formulation of cracks at constitutive points, $c p$, and smeared constitutive behavior obtained from interpolation between constitutive points at integration points, $i p$.

The hinge width $s$ is a fundamental calibration parameter of the model, and it was suggested in [19] to use a hinge width half the height of the beam, also adopted in the present study. Thus, the flexural deformation of the beam is concentrated and the propagation of a crack can be modelled as a hinge whereas the rest of the beam can be treated as elastic bulk material.

The uni-axial tensile behaviour of the cemented material is modelled according to the fictitious crack model by Hillerborg et al. [18]. The linear elastic pre-crack state is described by the elastic modulus, $E_{c}$. The uni-axial tensile strength is denoted by $f_{t}$ and the corresponding strain by $\varepsilon_{c t}$. To make the proposed model as versatile as possible, a multi-linear stress-crack opening relationship, or so-called softening law is selected 


$$
\sigma(w)=b_{i}-a_{i} w \begin{cases}b_{1}-a_{1} w & 0 \leq w \leq w_{1} \\ b_{2}-a_{2} w & w_{1} \leq w \leq w_{2} \\ b_{3}-a_{3} w & w_{2} \leq w \leq w_{3} \\ & \cdots \\ 0 & w_{i}>w_{c}\end{cases}
$$

where $w$ is the crack opening, $w_{c}$ is the final zero-stress displacement, $a_{i}$ is the slope and $b_{i}$ the intersection of the tangent line segment and the abscissa for a given point on softening curve as shown in Figure 2.

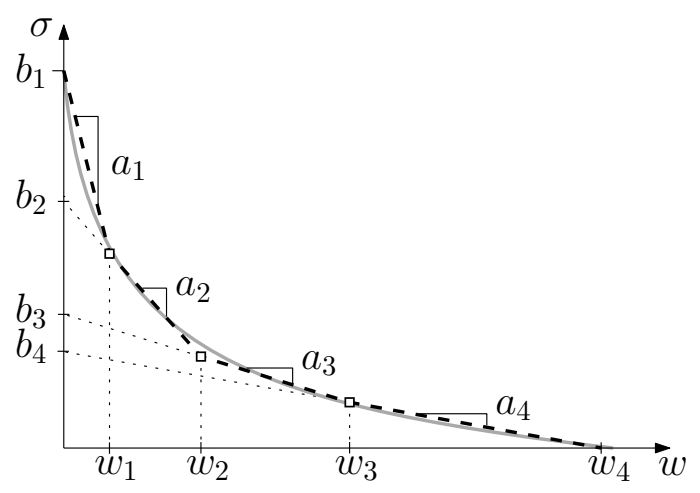

Figure 2: Definition of parameters $a_{i}, b_{i}$ and $w_{i}$ for softening law selected: multi-linear idealisation (black dashed) of exponential softening curve (gray solid) using four linear line segments.

The total fracture energy $G_{F}$ given by the area under the softening curve is

$$
G_{F}=\int_{0}^{w_{c}} \sigma(w) d w=\frac{1}{2} \sum_{i=1}^{n}\left[\left(2 b_{i}-a_{i}\left(w_{i-1}+w_{i}\right)\right)\left(w_{i}-w_{i-1}\right)\right]
$$

where $n$ is the number of lines on the softening curve.

For the semi-analytical hinge models published in the literature, see eg. [20, 25, 33, 26], sectional forces are calculated over the full beam-hinge segment (integration in blocks) for a given stress crack-opening relationship. A 
similar approach was used in the fnite element implementation of the crackedhinge reported by Olesen and Poulsen [23]. This type of model is simple and numerically robust. However, such formulation becomes cumbersome if one wish to study unloading- and reloading of cracks as well as more complex material behaviour, e.g. damage and fatigue. Following the basic idea of the 'multilayer model' by Hordijk [24], this study presents a general hinge model consisting of fibers of cemented material shown in Figure 3.

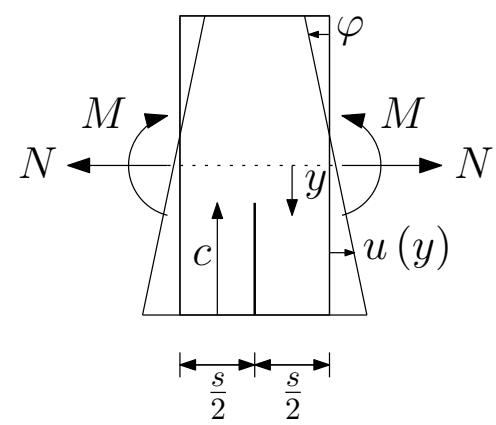

(a)

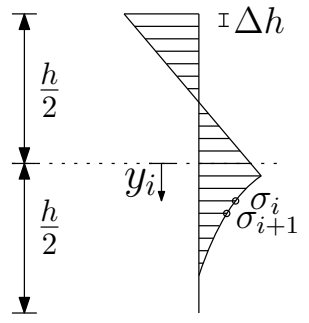

(b)

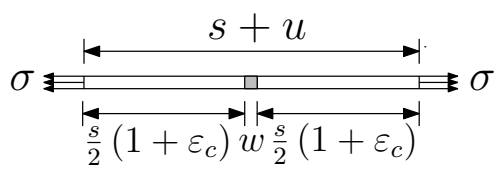

(c)

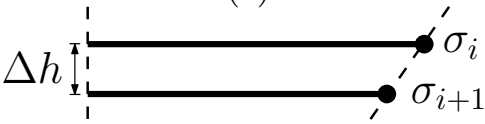

$(d)$

Figure 3: Fiber hinge model: (a) Beam segment with constant sectional forces and deformation of cracked beam segment. (b) Hinge stress distribution after initiation of cracking showing the individual fibers ( $n=24$, whereof 4 stress free). (c) Material fiber in uni-axial tension: loaded state beyond peak-load showing crack deformations. (d) Geometrical definition of one hinge strip (interpolation of stresses between two fibers).

The tensile behaviour of the hinge may be established by considering a fiber of material in uni-axial tension as shown in Figure 3 (c). The elongation of the fiber located at $y$ can be expressed in terms of the mean normal strain

$$
\bar{\varepsilon}(y)=\frac{2 u(y)}{s}=\frac{2 u_{0}(y)}{s}+\frac{2 \varphi}{s} y \equiv \bar{\varepsilon}_{0}+\bar{\kappa} y
$$

where $\bar{\varepsilon}_{0}$ is the mean normal strain at the beam axis, and $\bar{\kappa}$ the mean curvature of the hinge. In the cracked state, $0<w \leq w_{c}$, the crack opening and the corresponding stress in the strip is given as

$$
\left.\begin{array}{l}
\sigma_{c}=\sigma_{w} \Rightarrow \sigma_{c}=b_{i}+a_{i} w \\
s+2 u=s\left(1+\varepsilon_{c}\right)+w \Rightarrow s \bar{\varepsilon}(y)=s \varepsilon_{c}+w
\end{array}\right\} \Rightarrow\left\{\begin{array}{l}
w_{i}=s \frac{E_{c} \bar{\varepsilon}(y)-b_{i}}{E_{c}+a_{i} s} \\
\sigma_{i}=E_{c} \frac{b_{i}-a_{i} s \bar{\varepsilon}(y)}{E_{c}-a_{i} s}
\end{array}\right.
$$


In the cracked state, $w_{c} \leq w$, the crack is stress free, leading to the simple solution for the strip

$$
\left.\begin{array}{l}
w=s(1+\bar{\varepsilon}(y))-s \\
\sigma_{c}=0
\end{array}\right\} \Rightarrow\left\{\begin{array}{l}
w_{i}=s \bar{\varepsilon}(y) \\
\sigma_{i}=0
\end{array}\right.
$$

The hinge is divided in, $n+1$, number of fibers with the strip height $\Delta h$ between fibers, shown in Figure 3 (b). The position (top-down) of each fiber with respect to $y=0$ can be calculated as $y_{i}=-h / 2+\Delta h \cdot(i-1)$. The normal force contribution from each strip between two fibers is given by

$$
N_{i}=\frac{1}{2}\left(\sigma_{i}+\sigma_{i+1}\right) \Delta h t
$$

The eccentricity of the normal force for each strip is found from trapezoidal calculation and is given by

$$
e_{i}=\frac{1}{3} \frac{\sigma_{i}+2 \sigma_{i+1}}{\sigma_{i}+\sigma_{i+1}} \Delta h+y_{i}
$$

The moment contribution for each strip then yields

$$
M_{i}=N_{i} \cdot e_{i}
$$

The sectional forces with respect to $y=0$ is then a sum of the contribution from all, $n$, strips and may be calculated from

$$
\begin{aligned}
& N\left(\bar{\varepsilon}_{0}, \bar{\kappa}\right)=t \int_{-h / 2}^{h / 2} \sigma_{c} d y=\sum_{i=1}^{n} N_{i} \\
& M\left(\bar{\varepsilon}_{0}, \bar{\kappa}\right)=t \int_{-h / 2}^{h / 2} \sigma_{c} y d y=\sum_{i=1}^{n} M_{i}
\end{aligned}
$$


Convergence of the hinge is evaluated plotting the normalised error in moment $\beta$ between the original hinge model [19] and the proposed hinge, considering a simple linear softening law for different hinge mesh densities, $n$ : 2-100, shown in Figure 4 (b). It is observed that sufficient accuracy can be obtained with 30 fibers, however, little is gained by increasing the number of fibers from 10 to 30. Typical moment-curvature response is shown in Figure 4 (a). 

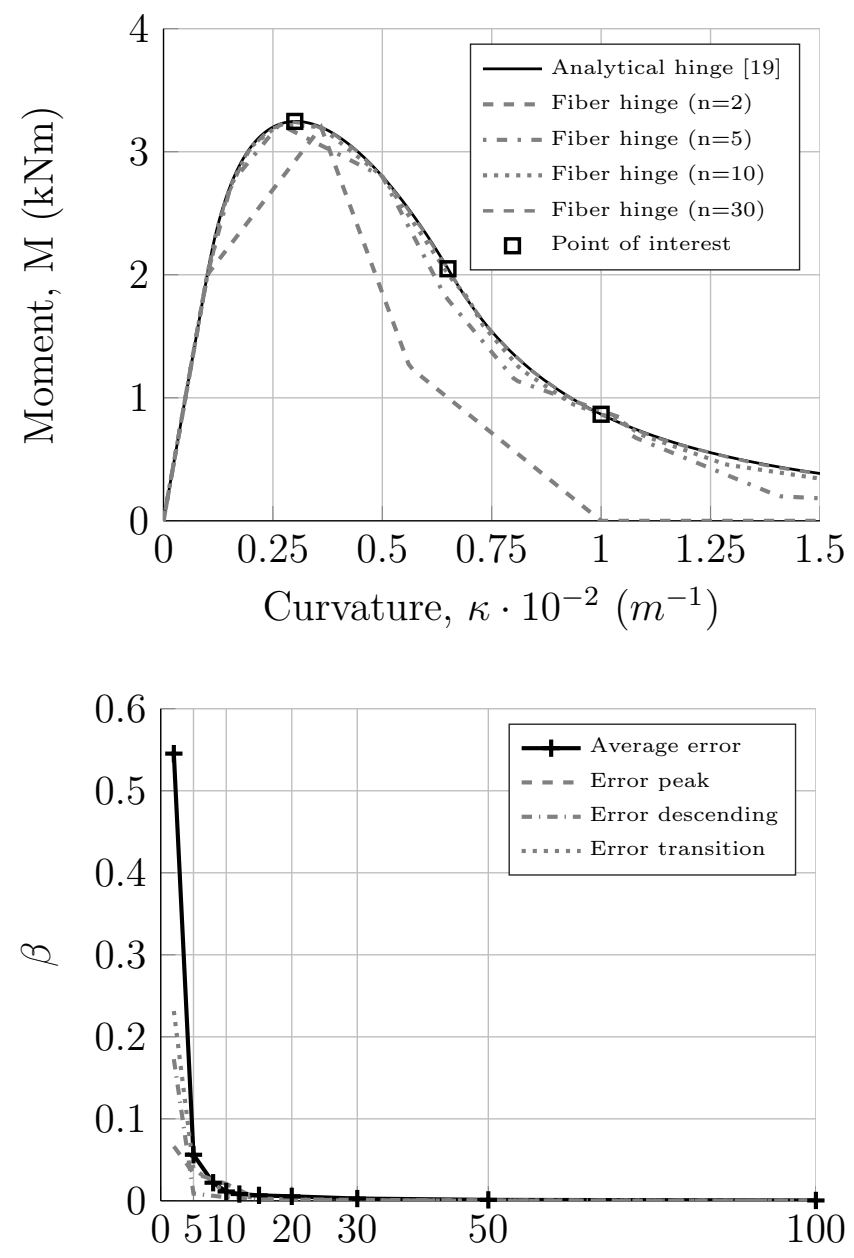

No. of fibers

Figure 4: (a) Typical moment-curvature response. (b) Number of hinge fibers, n: 2-100 (element size, elsz: 0.01-0.002 $\mathrm{m}$ ) versus the average normalised difference in moment $\beta$. Hinge dimensions $(\mathrm{h} / \mathrm{t}): 0.20 \times 0.10 \mathrm{~m}^{2}$. Material properties: $E_{c}=30 \mathrm{GPa}, f_{t}=3.5 \mathrm{MPa}$ $G_{F}=150 \mathrm{~N} / \mathrm{m}$ and $w_{c}=0.1 \mathrm{~mm}$ (linear softening).

In the analysis presented in Figure 4 the error in normalised moment is taken as the average difference in moment between the analytical hinge model in [19] and the proposed fiber hinge; at peak $\left(\kappa=0.003 \mathrm{~m}^{-1}\right)$, on the descending branch $\left(\kappa=0.0065 \mathrm{~m}^{-1}\right)$ and at the transition point between softening and stress free bottom fiber $\left(\kappa=0.01 \mathrm{~m}^{-1}\right)$. 


\section{Tensile damage model for cemented material}

In order to realistically capture the influence from unloading of a previously open crack, as well as the stiffness reduction for structures subjected to repeated loads, a low-cyclic damage plasticity model for the fiber in tension is implemented. A simple format is proposed, defining a fixed negative intersecting point $\sigma_{u}^{k}$ on the abscissa towards which unloading takes place after initiation of cracking. The geometrical meaning of the strain components for the damage-plasticity model are shown in Figure 5.

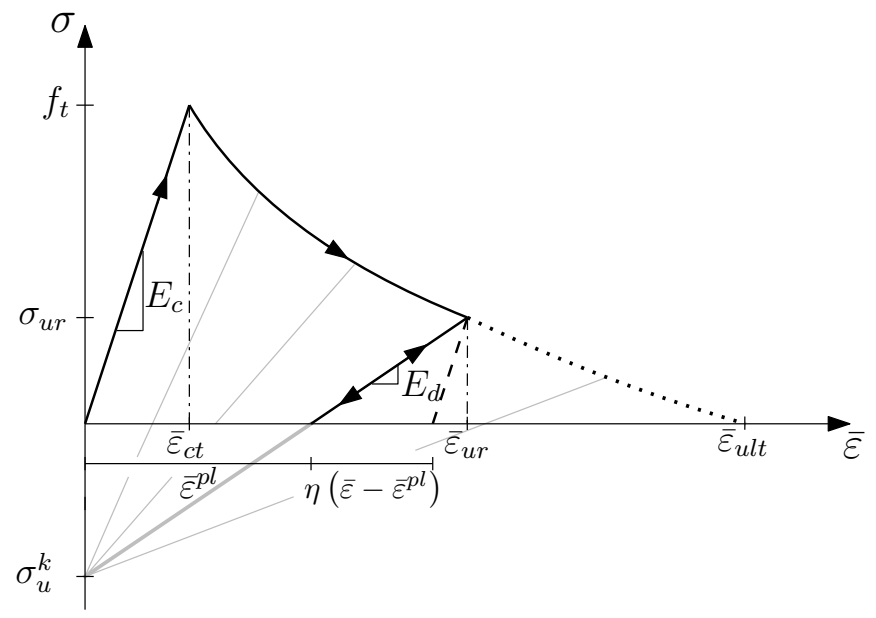

Figure 5: Geometrical meaning of strain components for the damage-plasticity model. The inelastic cracking strain $\bar{\varepsilon}^{c r}$ is composed of the reversible $\eta\left(\bar{\varepsilon}-\bar{\varepsilon}^{p l}\right)$ and irreversible $\bar{\varepsilon}^{p l}$ parts. The dashed line represent elastic unloading with the initial stiffness whereas grey lines represent the reduced stiffness and unloading towards point $\sigma_{u}^{k}$.

From Figure 5 it is evident that the unloading stiffness and plastic strain component can be defined as

$$
\begin{aligned}
& E_{s}^{ \pm}=\frac{\sigma_{u r}-\sigma_{u}^{k}}{\bar{\varepsilon}_{u r}} \\
& \bar{\varepsilon}^{p l}=-\frac{\sigma_{u}^{k}}{E_{s}^{ \pm}}
\end{aligned}
$$

where $E_{s}^{ \pm}$is the unloading- and reloading stiffness and $\sigma_{u r}$ and $\bar{\varepsilon}_{u r}$ is the maximum cracking stress and strain upon unloading and reloading, respectively. 
The format above is found to comply well with experimental data for concrete materials as well as more advanced concrete damage plasticity model formats, see e.g. [34, 35, 36]. Accordingly, a conventional 1-D damage-plastic stress strain law can be formulated

$$
\sigma=(1-\eta) E_{c}\left(\bar{\varepsilon}-\bar{\varepsilon}^{p l}\right)
$$

where the damage parameter is given as

$$
\eta=1-\frac{E_{s}^{ \pm}}{E_{c}}
$$

The crack width $w$ can be found from

$$
w= \begin{cases}s \bar{\varepsilon}-s \frac{\sigma_{u r}}{E_{c}} \Rightarrow s \bar{\varepsilon}^{c r} & 0<w \leq w_{c} \\ s \bar{\varepsilon} & w_{c}<w\end{cases}
$$

where the inelastic strain is given as $\bar{\varepsilon}^{c r}=\bar{\varepsilon}^{p l}+\eta\left(\bar{\varepsilon}-\bar{\varepsilon}^{p l}\right)$.

The constitutive behavior of a single hinge-fiber is tested plotting the stress-crack opening curve for one hinge fiber subjected to cyclic loading versus experimental results of plain concrete in uni-axial tension reported in Reinhardt et al. [37], shown in Figure 6. 


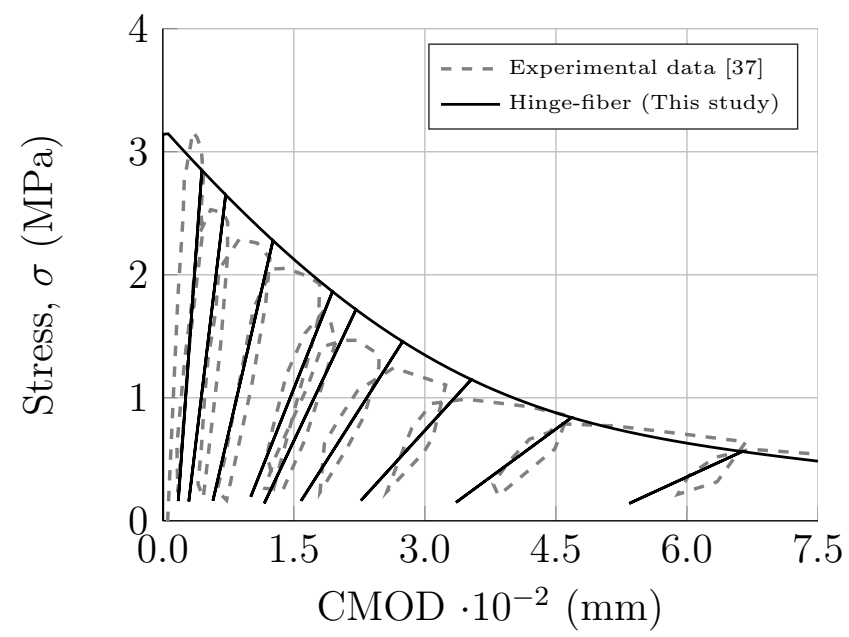

Figure 6: Constitutive behaviour of hinge-strip in uni-axial tension; stress-crack opening displacement compared to experimental results reported by Reinhardt et al. [37]. Relevant material parameters are; $E_{c}=39.27 \mathrm{GPa}, f_{t}=3.2, G_{F}=99.7 \mathrm{~N} / \mathrm{m}, c_{1}=3, c_{2}=6.93, w_{c}=$ $5.14 G_{F} / f_{t}=0.16 \mathrm{~mm}, s=0.25 \mathrm{~m}$ and $\sigma_{u}^{k}=-0.4 f_{t}$ (exponential softening).

It is observed from Figure 6 that good fit is obtained between the hingefiber behavior and experimental results. More detailed studies of the applicability of the semi-analytical cracked-hinge model can be found in [38].

\section{Implementation of hinge into beam element}

The proposed fiber hinge, in subsequent chapters referred to as the 'FEM hinge', is implemented in a user-built finite element code following standard finite element beam theory and procedures for building elements with nonlinear material behaviour, see e.g. [39]. The expressions for the element stiffness matrix and equivalent nodal loads is based on the cubic displacement function.

For the present study a plane three-node beam element is chosen as shown in Figure 7. This element is capable of modelling quadratic variations of the axial displacements and cubic variations of the transverse displacements. The choice of element ensures that both generalised strains are interpolated linearly as opposed to a typical two-node beam element where constant normal strain is assumed. 


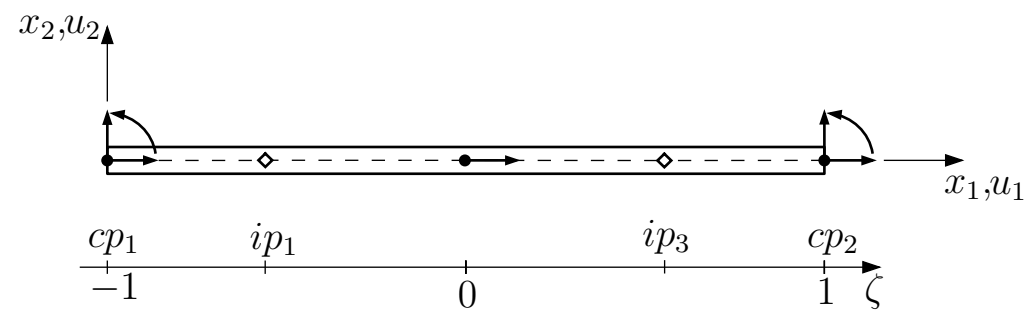

Figure 7: Plane beam element: Constitutive points $\left(c p_{i}\right)$ are located at endpoints, integration points $\left(i p_{i}\right)$ at Gauss points $\pm \sqrt{1 / 3}$.

The vector of generalised strains, $\boldsymbol{\varepsilon}$, holds the linearised axial strain $\varepsilon_{0}$ and the linearised curvature $\kappa$. The interpolation of $\varepsilon$ in the element is given by

$$
\boldsymbol{\varepsilon}=\left[\begin{array}{c}
\varepsilon_{0} \\
\kappa
\end{array}\right]=\left[\begin{array}{c}
\frac{d u_{1}}{d x} \\
\frac{d^{2} u_{2}}{d x^{2}}
\end{array}\right]=\mathbf{B} \boldsymbol{v}
$$

where $\mathbf{B}$ is the strain interpolation matrix. The vector of generalised stresses, $\boldsymbol{\sigma}$, holds the sectional normal force $N$ and the sectional moment $M$ and may be established applying (9a) and (9b), i.e. $\boldsymbol{\sigma}=\boldsymbol{\sigma}(\boldsymbol{\varepsilon})=\left[\begin{array}{l}N(\boldsymbol{\varepsilon}) \\ M(\boldsymbol{\varepsilon})\end{array}\right]$.

The hinge model presented here first determine the constitutive state and stiffness of each individual fiber. Integration over the strip height between fibers is then performed and the sum of all contributions is included in the tangent stiffness matrix. The hinge tangent stiffness matrix, $\mathbf{D}_{t}$ is defined through

$$
\left[\begin{array}{l}
d N \\
d M
\end{array}\right]=\mathbf{D}_{t}\left[\begin{array}{l}
d \bar{\varepsilon}_{0} \\
d \bar{\kappa}
\end{array}\right] \quad, \quad \mathbf{D}_{t}=\sum_{i=1}^{n}\left[\begin{array}{cc}
\frac{\partial N_{i}}{\partial \bar{\varepsilon}_{0}} & \frac{\partial N_{i}}{\partial \bar{\kappa}} \\
\frac{\partial M_{i}}{\partial \bar{\varepsilon}_{0}} & \frac{\partial M_{i}}{\partial \bar{\kappa}}
\end{array}\right]
$$

Monotonic loading of the hinge results in constant positive stiffness of fibers in linear elastic state. Fibers in the cracked state along the softening branch and cracked stress-free state result in negative and zero stiffness contributions, respectively. The constituents of (15) are obtained from (9a) and (9b) utilising the following relations for the relevant part of the integral corresponding to $0<w \leq w_{c}$ 


$$
\frac{\partial \sigma_{c}}{\partial \bar{\varepsilon}_{0}}=E_{c} \frac{a_{i} s}{E_{c}+a_{i} s} \quad, \quad \frac{\partial \sigma_{c}}{\partial \bar{\kappa}}=E_{c} \frac{a_{i} s}{E_{c}+a_{i} s} y
$$

Here the parameters $\alpha_{i}=\frac{a_{i} s}{E_{c}+a_{i} s}$ and $E_{c c}^{i}=E_{c} \alpha_{i}$ are introduced, where the latter symbolises the reduced stiffness of the cracked fiber. $a_{i}$ is the slope at a given point on the softening curve, see Figure 2. The stiffness contribution from one fiber in the three different phases; elastic, softening and stress-free is given by $(17 \mathrm{a}),(17 \mathrm{~b})$ and $(17 \mathrm{c})$, respectively

$$
\begin{array}{rlr}
\mathbf{D}_{t}^{e l} & =\left[\begin{array}{ll}
E_{c} t & E_{c} t y \\
E_{c} t y & E_{c} t y^{2}
\end{array}\right] & \bar{\varepsilon} \leq \bar{\varepsilon}_{e c t} \\
\mathbf{D}_{t}^{c r} & =\left[\begin{array}{ll}
E_{c c} t & E_{c c} t y \\
E_{c c} t y & E_{c c} t y^{2}
\end{array}\right] & \bar{\varepsilon}_{\text {ect }}<\bar{\varepsilon} \leq \bar{\varepsilon}_{\text {ult }} \\
\mathbf{D}_{t}^{0} & =\left[\begin{array}{ll}
0 & 0 \\
0 & 0
\end{array}\right] & \bar{\varepsilon}>\bar{\varepsilon}_{\text {ult }}
\end{array}
$$

In the case of unloading- and reloading, a fiber in linear elastic state will not change whereas a fiber along the softening branch change from a negative to a positive stiffness contribution. Moreover, the tensile damage parameter is introduced, i.e.

$$
\mathbf{D}_{t}^{u}=(1-\eta) \mathbf{D}_{t}^{e l} \quad \text { for } \quad \bar{\varepsilon}_{c t}<\bar{\varepsilon}^{u n l}<\bar{\varepsilon}_{u l t}
$$

The full tangent stiffness matrix for loading, unloading and reloading can now be established by interpolation between each fiber and integration over the strip height as given in Appendix A.1. The internal nodal force and the contribution from the beam-element to the tangential stiffness matrix can then be found from standard finite element beam theory. 


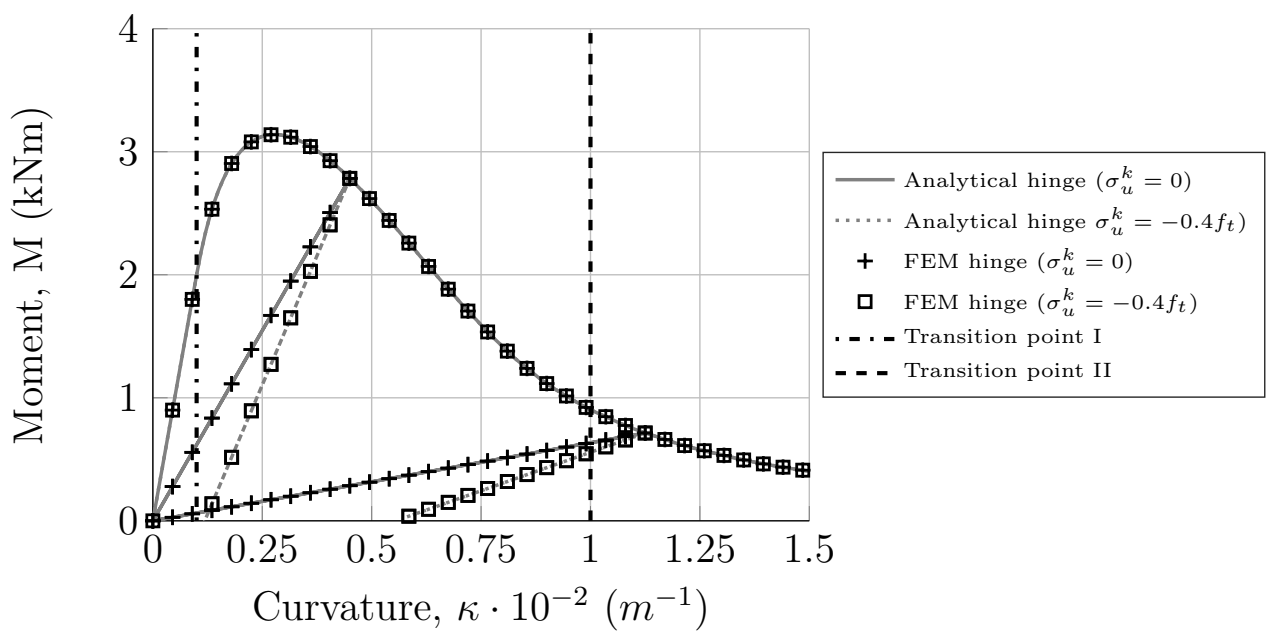

Figure 8: Implementation of hinge into beam element: comparison between analytical and finite element hinge model. Hinge dimensions $(\mathrm{h} / \mathrm{t}): 0.20 \times 0.10 \mathrm{~m}^{2}$. Material properties: $E_{c}=30 \mathrm{GPa}, f_{t}=3.5 \mathrm{MPa}, G_{F}=150 \mathrm{~N} / \mathrm{m}$ and $w_{c}=0.1 \mathrm{~mm}$ (linear softening).

The implemented hinge is validated by plotting the moment-curvature behavior for a single FEM hinge versus the analytical hinge. It can be observed from Figure 8 that exact fit is obtained between the FEM hinge and analytical model considering two types of unloading schemes. Fast convergence, within 1-2 iterations, is obtained for this simple model applying a fixed increment of $\Delta \theta=0.225 \mathrm{~mm}^{-1}$. The two transition points between the phases; elastic-softening (crack initiation) and softening-stress free (bottom fiber stress free) are shown for reference.

\section{Structural examples}

\subsection{Finite element model}

The performance of the proposed hinge model is further evaluated by structural analysis of two fracture tests on plain concrete. First, a conventional notched beam under three point loading is considered, shown in Figure 9 (a). Second, a notched beam resting on clay soil subjected to a concentrated load at midspan position is considered, shown in Figure 9 (b). Beam geometry, material properties and model parameters used in the analysis are given in Table 1. Overview of model geometry and boundary conditions is shown in Figure 1. 
Table 1: Geometry and mechanical properties for beams used in numerical studies.

\begin{tabular}{|c|c|c|c|}
\hline Geometry & Unit & Three-point & Soil \\
\hline Length, $L$ & $(\mathrm{~m})$ & 0.60 & 0.80 \\
\hline Height, $H$ & $(\mathrm{~m})$ & 0.15 & 0.15 \\
\hline Thickness, $t$ & $(\mathrm{~m})$ & 0.08 & 0.08 \\
\hline Notch depth, $a_{0}$ & $(\mathrm{~m})$ & 0.05 & 0.05 \\
\hline \multicolumn{4}{|l|}{ Mechanical- and fracture properties } \\
\hline Young's modulus, $E$ & $(\mathrm{MPa})$ & 32,040 & 32,040 \\
\hline Poisson's rato, $v$ & $(-)$ & 0.15 & 0.15 \\
\hline Tensile strength, $f_{t}$ & $(\mathrm{MPa})$ & 4.15 & 4.15 \\
\hline Fracture energy, $G_{F}$ & $(\mathrm{~N} / \mathrm{mm})$ & 0.164 & 0.164 \\
\hline \multicolumn{4}{|l|}{ Soil properties } \\
\hline Vertical spring, $k_{v}$ & $(\mathrm{MPa} / \mathrm{mm})$ & - & 0.1560 \\
\hline Horisontal spring, $k_{h}$ & $(\mathrm{MPa} / \mathrm{mm})$ & - & 0.0156 \\
\hline \multicolumn{4}{|l|}{ Cohesive model parameters } \\
\hline Softening type & $(-)$ & Bilinear & Bilinear \\
\hline Stress ratio at kink point, $k_{1}$ & $(-)$ & 0.25 & 0.25 \\
\hline Displacement at kink point, $w_{k_{1}}$ & $(\mathrm{~mm})$ & 0.0204 & 0.0204 \\
\hline Final zero displacement, $w_{c}$ & $(\mathrm{~mm})$ & 0.234 & 0.234 \\
\hline \multicolumn{4}{|c|}{ Hinge model and numerical parameters } \\
\hline Number of fibers, $n$ & $(-)$ & 30 & 30 \\
\hline Number of elements, nel & $(-)$ & 8 & 10 \\
\hline Unloading point, $\sigma_{u}^{k}$ & $(\mathrm{MPa})$ & $-0.4 f_{t}$ & $-0.4 f_{t}$ \\
\hline Increment size, $\Delta \delta_{c}$ & $(\mathrm{~mm})$ & $1 \cdot 10^{-2}$ & $1 \cdot 10^{-2}$ \\
\hline Error tolerance, $\epsilon$ & $(-)$ & $1 \cdot 10^{-4}$ & $1 \cdot 10^{-4}$ \\
\hline
\end{tabular}

To simulate the softening response of beam structures and to compare experimental- and numerical unloading and reloading curves, a conventional Newton-Raphson (N-R) solver is implemented with direct displacement control, see e.g. [40]. In the present study the energy norm ratio $\delta E_{1} / \Delta E_{0}$ is applied as a measure in the convergence criterion. 
(a)

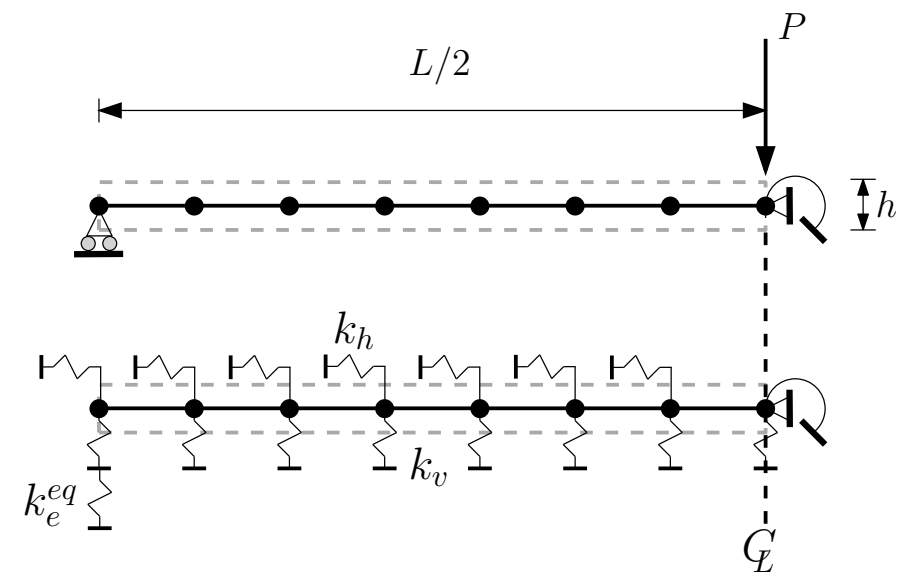

Figure 9: Model geometry and boundary conditions of finite element model used in the present study: (a) simply supported beam and (b) soil supported beam.

\subsection{Three-point beam}

Convergence of the FEM hinge model is evaluated plotting the load displacement behavior of a three point-beam, see Figure 9 (a), for different beam mesh densities, nel: 2-14, shown in Figure 10. It is observed that sufficient accuracy for peak-load and peak-load displacement can be obtained with only 2 elements. However, on the softening branch, a minimum of 8 elements are needed to resemble the softening response of the analytical model. To obtain satisfactory convergence a minimum of 10 elements are needed, resulting in an element size of $0.06 \mathrm{~m}$, selected in subsequent analysis. In the analytical model, implementing the hinge into an elastic beam as suggested by Olesen [20], only one hinge is considered. This explains the slightly stiffer behavior compared to the finite element hinge. 


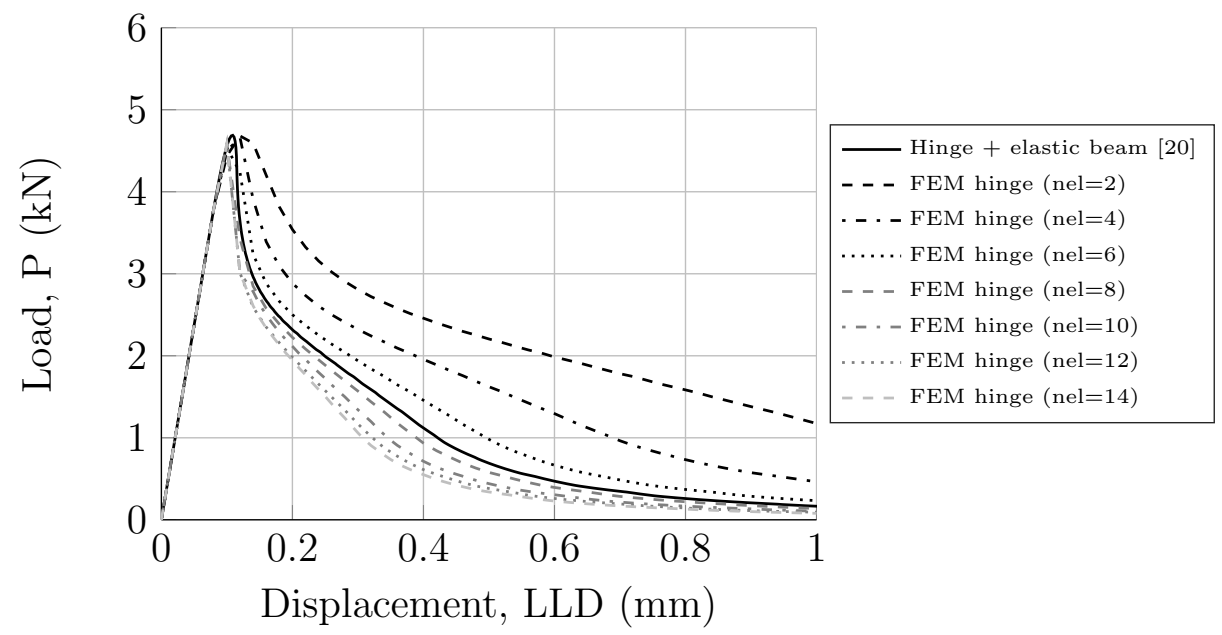

Figure 10: Convergence test of simply supported showing typical load line displacement (LLD) response for different mesh densities nel: 2-14 of the FEM hinge compared to the analytical model considering one crack only.

The functionality of the proposed numerical hinge to simulate the lowcycle fracture behavior of a three-point beam is demonstrated by plotting the load-crack mouth opening displacement versus experimental and numerical results reported in [16], see Figure 11 (a) and (b), respectively. 

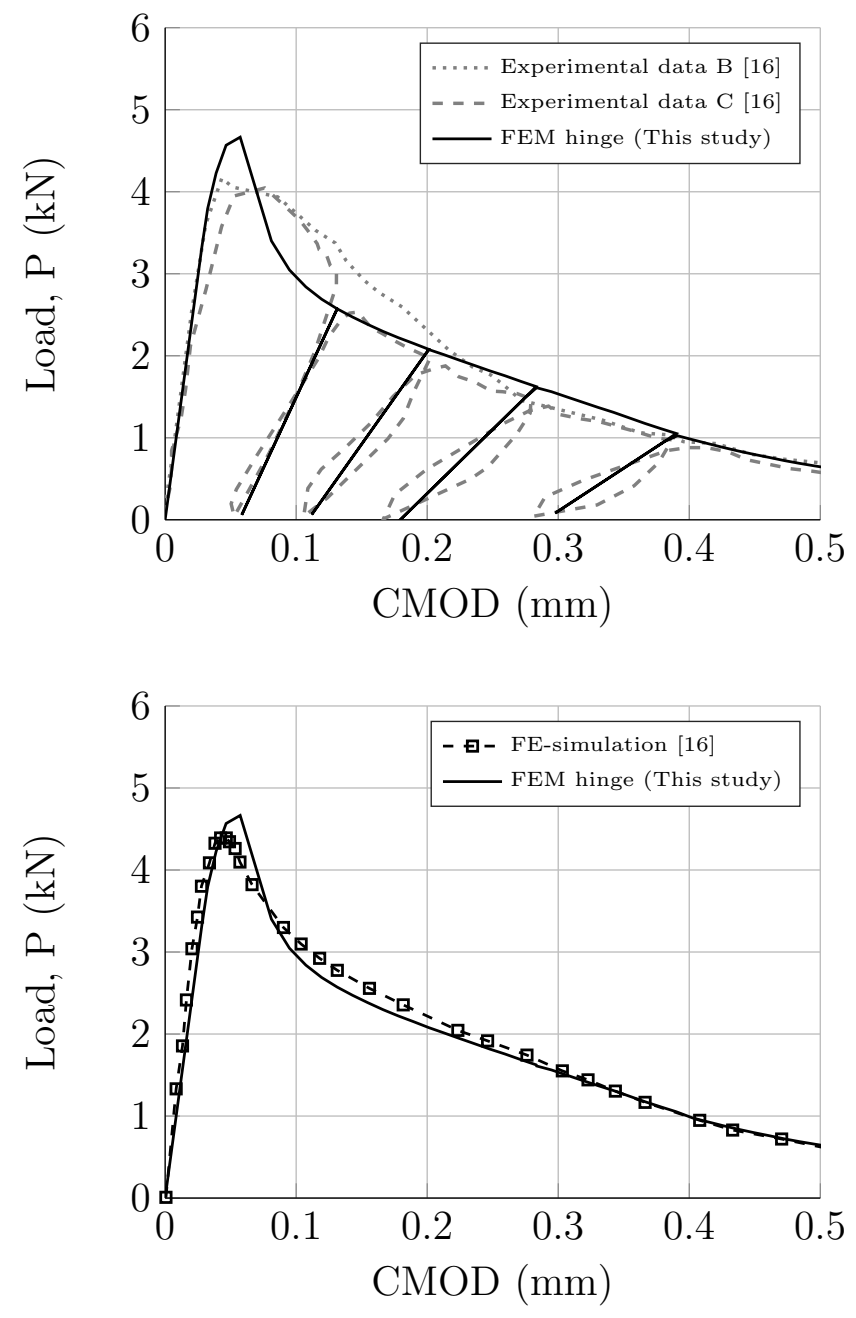

Figure 11: Load-crack mouth opening displacement response of a three-point beam: (a) FEM hinge versus experimental curves plotting 4 of 19 load cycles carried out in experiment. (b) FEM hinge and analytical hinge versus a discrete cohesive zone model.

It is observed from the load-CMOD curve in Figure 11 (a) that there is good agreement between the FEM hinge model and the experimental results. The hinge model resemble both pre-peak, softening and unloading- reloading response adequately well. The difference in peak-load and peak-load displacement is app. $10 \%$ and $5 \%$ respectively, which is within the expected scatter. It is also found that the monotonic behavior of the proposed hinge comply well with finite element simulations using a discrete cohesive zone 
model and the commercial computer package ABAQUS reported in [16], see Figure $11(\mathrm{~b})$.

The difference between the FEM hinge and the cohesive zone model is mainly related to the pre-peak behavior. Whereas the hinge model behaves perfectly elastic up to initiation of cracking, the cohesive zone model incorporates a small error in crack-opening displacements related to the penalty stiffness. Increasing penalty stiffness reduce this error, however, very high levels of penalty stiffness result in ill-conditioned stiffness matrix and thus slow convergence and aborted simulations. This problem is avoided applying the proposed hinge, resulting in a more robust model and stable simulations.

\subsection{Beam resting on soil foundation}

The beam resting on soil foundation is modeled using a simple spring soil model suggested by Gaedicke and Roesler [16]. The idealised spring foundation model consists of independent vertical springs with a spring stiffness coefficient, $k_{v}$, similar to a conventional Winkler model. The constitutive behavior of the vertical spring is modified to allow for separation between the beam and soil, i.e. for positive vertical displacements (tensionless spring). Moreover, the horisontal spring is implemented in the model, representing the frictional contact between soil and beam structure. The horisontal spring is modeled with a stiffness of $k_{h}=k_{v} / 10$ and a constant slip limit of $u_{h}=0.5$ $\mathrm{mm}$. A schematic overview of the spring constitutive behavior is shown in Figure 12. Implementation of the Winkler foundation model into beam elements is described in Appendix A.2.

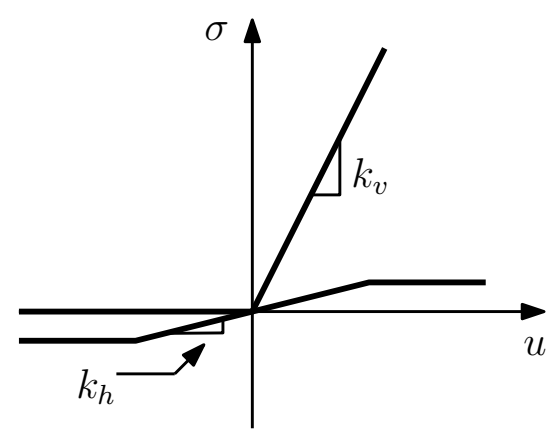

Figure 12: Constitutive behavior of Winkler spring foundation applied in [16]. 
The functionality of the proposed numerical hinge to simulate the lowcylic fracture behavior of a beam resting on soil is demonstrated by plotting the load-crack mouth opening displacement versus experimental- and numerical results reported in [16], see Figure 13 (a) and (b), respectively
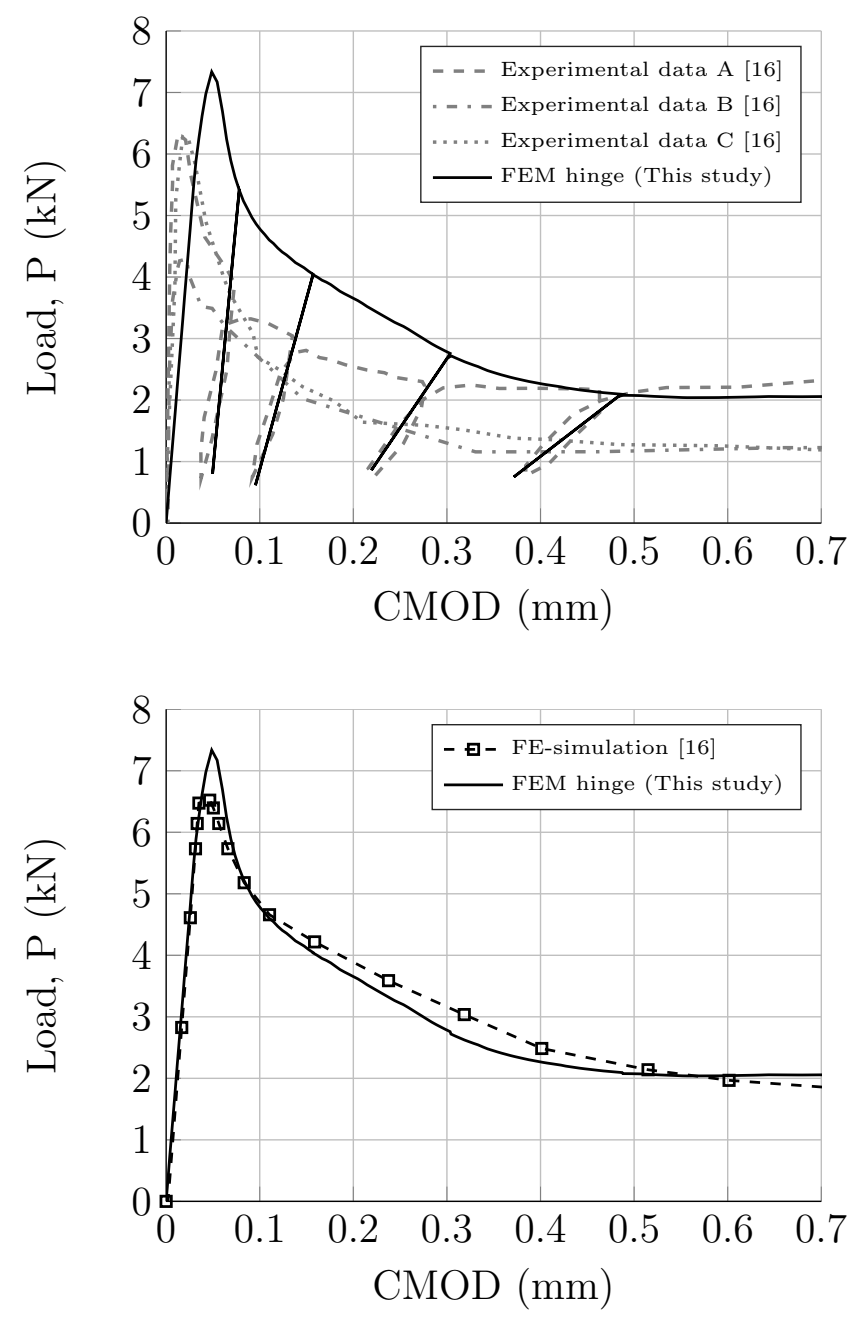

Figure 13: Load-crack mouth opening displacement response of beam on soil: (a) FEM hinge versus experimental curves plotting 4 of 18 load cycles carried out in experiment. (b) FEM hinge versus a discrete cohesive zone model.

It is observed from the load-CMOD curve in Figure 13 (a) that the FEM hinge model captures the overall characteristic response of the beam on soil 
on the descending branch. However, the peak-load and load level in general, as well as the peak-displacement is overestimated. The difference in peak-load varies between 15-50\% compared to experimental curves. Similar trend is also found in numerical analysis reported in [16]. Thus, the FEM hinge comply well with the numerical results applying a discrete cohesive zone model as shown in Figure 13 (b). In the example presented, the convergence rate is relatively fast, and below 10 iterations, during unloading and reloading. The convergence rate during monotonic post-peak loading is somewhat slower. This is can be explained by the sudden change in stiffness at peak and at the point where the reloading curve enters the monotonic curve.

The difference between experimental and numerical results indicate that the soil model applied in simulations does not reflect the stress distribution below the beam and/or other boundary conditions in the test set-up very well. The stiff pre-peak response and lower peak load in experiments indicate that the continuity and shear stiffness of the soil influence the response significantly. For cohesive soils, considered here, this influence will theoretically result in a large vertical reaction pressure along beam edges.

In order to obtain a more realistic response of the soil a generalised twoparameter spring model, see e.g. [41, 38], is implemented in beam elements as shown in Appendix A.2. The two-parameter model introduce an interaction between independent springs through a shear coupling spring, $\gamma$. A simple approach is applied here to demonstrate the applicability of the twoparameter model keeping the Winkler stiffness constant and then adjusting the second parameter to match the pre-peak behavior of experimental beams, resulting in $\gamma=1.75 \cdot 10^{6} \mathrm{~N}$. The soil adjacent to the beam is modeled using an additional spring force, i.e., $k_{e}^{e q}=k_{v} \sqrt{\gamma / k_{v}}$. 


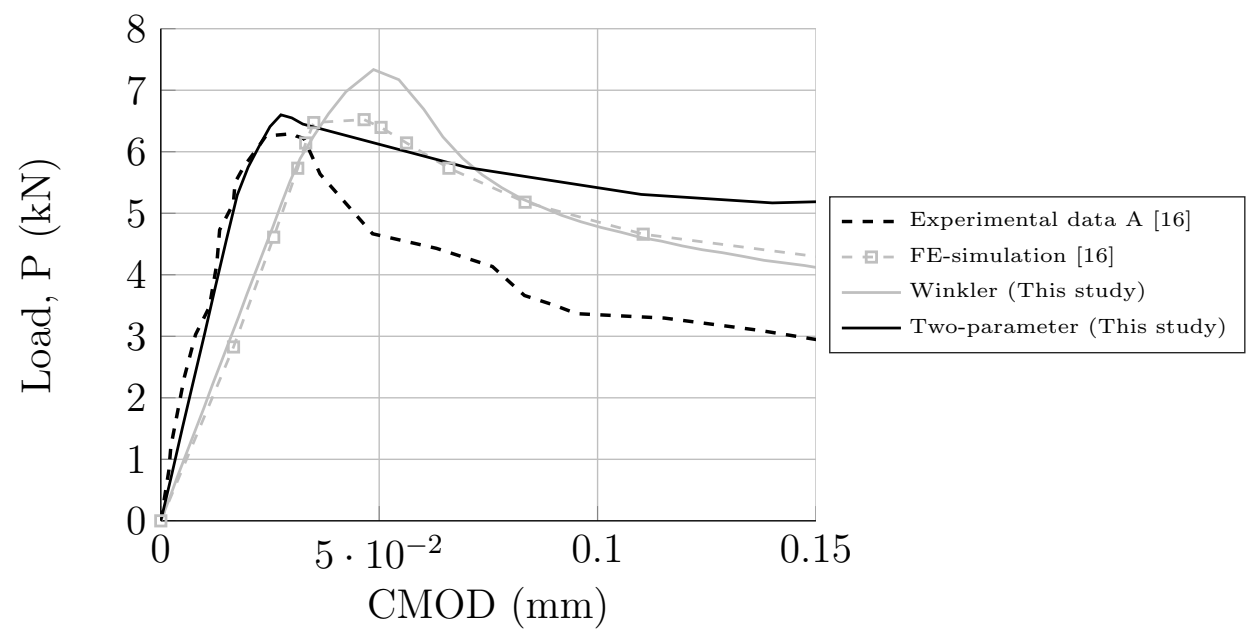

Figure 14: Comparison between experimental results (gray dashed), numerical results applying a Winkler model (black solid) and numerical results applying a calibrated twoparameter model (gray solid): Load-crack mouth opening displacement response of three point beam on soil.

It is observed from Figure 14 that the peak-load and peak-load displacement can be predicted adequately well applying a two-parameter model. However, on the post-peak branch, the continuity created by the second parameter spring, result in a high residual stiffness of the structure. At this stage the shallow clay soil layer is likely to exhibit severe plastic yielding due to the stress concentration at the crack front. To obtain a realistic response for the total load-CMOD curve, it would be necessary apply more advanced foundation models, e.g. by extending the two-parameter to account for plastic yielding.

\section{Conclusion}

The use of a cohesive cracked-hinge model for simulating low-cyclic damage of cemented beam structures has been investigated showing good performance. Implementation of the hinge into a beam element is relatively straightforward and the contribution to the tangent stiffness matrix from each fiber is established following a general format, creating a versatile tool, allowing for different types of softening laws and damage formats.

The analytical hinge model developed shows satisfying performance compared to integration over the full hinge segment. Good fit is obtained using 
a relatively coarse discretisation of 10-30 fibers. Moreover, it is found that the analytical damage-plasticity format implemented sufficiently captures the response of experimental cyclic uni-axial tensile tests.

The results obtained show that the proposed numerical hinge model adequately describe the cyclic response of a plain concrete beam under three point loading. It is also found that the proposed hinge comply well with other numerical results published in the literature, applying a cohesive zone model using the same cohesive model parameters.

Simulation of beams resting on soil foundation show that the hinge model captures the main structural response of experimental beams. The hinge model resting on Winkler foundation does not describe the pre-peak behavior observed in experiments adequately well, indicating that the soil model and the boundary conditions applied does not comply with the test set-up. This highlights the influence from soil-structure interaction on the structural response of beams on soil. To overcome the deficiency of the Winkler model, an interaction between independent springs is introduced, using a two-parameter spring model, showing good performance.

The present paper demonstrates the implementation and application of a general cohesive crack-hinge model to describe the fracture behavior of cemented beam structures. The results obtained is encouraging and show that the methodology is well suited for practical use.

\section{Acknowledgements}

This work was supported by COWIfonden. 


\section{References}

[1] R. G. Packard, Thickness design for concrete highway and street pavements, Portland Cement Association.

[2] NCHRP, Guide for mechanistic-empirical design of new and rehabilitated pavement structures: Final report for project 1-37a, National Cooperative Highway Research Program, Transportation Research Board, National Research Council, Washington, DC.

[3] J. Knapton, The Structural Design of Heavy Duty Pavements for Ports and Other Industries, Interpave, 4th Edition (December 2008).

[4] Y.-S. Jenq, J.-D. Perng, Analysis of crack propagation in asphalt concrete using cohesive crack model, Transportation Research Record: Journal of the Transportation Research Board (1317) (1991) 90-99.

[5] A. Scarpas, R. Al-Khoury, C. Van Gurp, S. Erkens, Finite element simulation of damage development in asphalt concrete pavements, Proceedings of the International Conference on Asphalt Pavements 1 (1997) 673-692.

[6] C. S. Desai, Unified dsc constitutive model for pavement materials with numerical implementation, International Journal of Geomechanics 7 (2) (2007) 83-101. doi:10.1061/(ASCE)1532-3641(2007)7:2(83).

[7] J. Baek, I. Al-Qadi, Finite element method modeling of reflective cracking initiation and propagation: Investigation of the effect of steel reinforcement interlayer on retarding reflective cracking in hot-mix asphalt overlay, Transportation Research Record: Journal of the Transportation Research Board (1949) (2006) 32-42.

[8] R. Bonaquist, M. Witczak, A comprehensive constitutive model for granular materials in flexible pavement structures, Proceedings of the International Conference on Asphalt Pavements 1 (1997) 783-802.

[9] T. Habiballah, C. Chazallon, An elastoplastic model based on the shakedown concept for flexible pavements unbound granular materials, International Journal for Numerical and Analytical Methods in Geomechanics 29 (6) (2005) 577-596. 
[10] D. D. Cortes, H. Shin, J. C. Santamarina, Numerical simulation of inverted pavement systems, Journal of Transportation Engineering-ASCE 138 (12) (2012) 1507-1519. doi:10.1061/(ASCE)TE.1943-5436.0000472.

[11] A. Meda, G. A. Plizzari, P. Riva, Fracture behavior of sfrc slabs on grade, Materials and StructuresS 37 (270) (2004) 405-411. doi:10.1617/14093.

[12] A. M. Ioannides, J. Peng, J. R. Swindler Jr, Abaqus model for pcc slab cracking, International Journal of Pavement Engineering 7 (4) (2006) 311-321.

[13] C. Gaedicke, J. Roesler, F. Evangelista, Three-dimensional cohesive crack model prediction of the flexural capacity of concrete slabs on soil, Engineering Fracture Mechanics 94 (2012) 1-12.

[14] J. Evangelista, Francisco, J. R. Roesler, S. P. Proena, Three-dimensional cohesive zone model for fracture of cementitious materials based on the thermodynamics of irreversible processes, Engineering Fracture Mechanics 97 (2013) 261-280.

[15] A. Skar, P. N. Poulsen, 3-d cohesive finite element model for application in structural analysis of heavy duty composite pavements, Construction and Building Materials 101, Part 1 (2015) 417 - 431. doi:http://dx.doi.org/10.1016/j.conbuildmat.2015.10.052.

[16] C. Gaedicke, J. Roesler, Fracture-based method to determine the flexural load capacity of concrete slabs, FAA COE Rep (31).

[17] C. Gaedicke, J. Roesler, Fracture-based method to determine flexural capacity of concrete beams on soil, Road materials and pavement design 11 (2) (2010) 361-385.

[18] A. Hillerborg, M. Modéer, P.-E. Petersson, Analysis of crack formation and crack growth in concrete by means of fracture mechanics and finite elements, Cement and concrete research 6 (6) (1976) 773-781.

[19] J. P. Ulfkjær, S. Krenk, R. Brincker, Analytical model for fictitious crack propagation in concrete beams, Journal of Engineering Mechanics 121 (1) (1995) 7-15. 
[20] J. F. Olesen, Fictitious crack propagation in fiber-reinforced concrete beams, Journal of Engineering Mechanics 127 (3) (2001) 272-280. doi:10.1061/(ASCE)0733-9399(2001)127:3(272).

[21] R. Walter, L. Østergaard, J. F. Olesen, H. Stang, Wedge splitting test for a steel-concrete interface, Engineering Fracture Mechanics 72 (17) (2005) 2565-2583.

[22] J. F. Olesen, L. Østergaard, H. Stang, Nonlinear fracture mechanics and plasticity of the split cylinder test, Materials and structures 39 (4) (2006) 421-432.

[23] J. F. Olesen, P. N. Poulsen, Modeling rc beam structures based on cracked hinge model and finite elements, Tech. Rep. SR12-11, Technical University of Denmark, DTU Civil Engineering (2012).

[24] D. Hordijk, Tensile and tensile fatigue behaviour of concrete; experiments, modelling and analyses, Heron 37 (1) (1992) 1-79.

[25] J. Zhang, V. C. Li, H. Stang, Size effect on fatigue in bending of concrete, Journal of materials in civil engineering 13 (6) (2001) 446-453.

[26] N. A. Brake, K. Chatti, Prediction of size effect and non-linear crack growth in plain concrete under fatigue loading, Engineering Fracture Mechanics 109 (2013) 169-185.

[27] A. Carpinteri, Energy dissipation in rc beams under cyclic loadings, Engineering Fracture Mechanics 39 (2) (1991) 177-184.

[28] J. Zhang, H. Stang, V. C. Li, Fatigue life prediction of fiber reinforced concrete under flexural load, International Journal of Fatigue 21 (10) (1999) 1033-1049.

[29] A. Carpinteri, A. Spagnoli, S. Vantadori, A fracture mechanics model for a composite beam with multiple reinforcements under cyclic bending, International Journal of Solids and Structures 41 (20) (2004) 5499-5515.

[30] A. Carpinteri, A. Spagnoli, S. Vantadori, An elastic-plastic crack bridging model for brittle-matrix fibrous composite beams under cyclic loading, International Journal of Solids and Structures 43 (16) (2006) 49174936. 
[31] S. R. Maitra, K. S. Reddy, L. S. Ramachandra, Numerical investigation of fatigue characteristics of concrete pavement, International Journal of Solids Fracture 189 (2) (2014) 181-193. doi:10.1007/s10704-014-9969-x.

[32] J. Planas, M. Elices, Nonlinear fracture of cohesive materials, in: Current Trends in Concrete Fracture Research, Springer, 1991, pp. 139-157.

[33] K. S. R. Iyengar, S. Raviraj, T. Jayaram, Analysis of crack propagation in strain-softening beams, Engineering Fracture Mechanics 69 (6) (2002) 761-778.

[34] K. Maekawa, J. Takemura, P. Irawan, M. Irie, Triaxial elastoplastic and fracture model for concrete, Doboku Gakkai RombunHokokushu/Proceedings of the Japan Society of Civil Engineers (460 pt 5-18) (1993) 131-138.

[35] J. Lee, G. Fenves, Plastic-damage model for cyclic loading of concrete structures, Journal of Engineering Mechanics 124 (8) (1998) 892-900.

[36] P. Grassl, D. Xenos, U. Nyström, R. Rempling, K. Gylltoft, Cdpm2: A damage-plasticity approach to modelling the failure of concrete, International Journal of Solids and Structures 50 (24) (2013) 3805-3816.

[37] H. W. Reinhardt, H. A. Cornelissen, D. A. Hordijk, Tensile tests and failure analysis of concrete, Journal of Structural Engineering 112 (11) (1986) 2462-2477.

[38] A. Skar, Deterioration models for cement bound materials in structural design and evaluation of heavy duty pavements, Ph.D. thesis, Technical University of Denmark (October 2016).

[39] R. D. Cook, et al., Concepts and applications of finite element analysis, John Wiley \& Sons, 2007.

[40] J.-L. Batoz, G. Dhatt, Incremental displacement algorithms for nonlinear problems, International Journal for Numerical Methods in Engineering 14 (8) (1979) 1262-1267.

[41] F. Zhaohua, R. D. Cook, Beam elements on two-parameter elastic foundations, Journal of Engineering Mechanics 109 (6) (1983) 1390-1402. 


\section{Appendix A. Appendix}

Appendix A.1. Derivation of hinge tangent stiffness matrix

Interpolation between hinge fibers and integration over the strip height is given as

$$
\begin{aligned}
d N_{i}= & t \int_{y_{i}}^{y_{i+1}} \frac{1}{2}\left(D_{i}(1,1)+D_{i+1}(1,1)\right) d y+\int_{y_{i}}^{y_{i+1}} \frac{1}{2}\left(D_{i}(1,2)+D_{i+1}(1,2)\right) d y \\
= & \frac{1}{2}\left(D_{i}(1,1)+D_{i+1}(1,1)\right)\left(y_{i+1}-y_{i}\right) \\
& +\frac{1}{2}\left(D_{i}(1,2)+D_{i+1}(1,2)\right)\left(y_{i+1}-y_{i}\right) \frac{1}{2}\left(y_{i}+y_{i+1}\right) \\
= & D_{i}(1,1) d \bar{\varepsilon}_{0}+D_{i}(1,2) d \bar{\kappa} \\
= & \frac{\partial N_{i}}{\partial \bar{\varepsilon}_{0}}+\frac{\partial N_{i}}{\partial \bar{\kappa}} \\
d M_{i}= & t \int_{y_{i}}^{y_{i+1}} \frac{1}{2}\left(D_{i}(2,1)+D_{i+1}(2,1)\right) d y+\int_{y_{i}}^{y_{i+1}} \frac{1}{2}\left(D_{i}(2,2)+D_{i+1}(2,2)\right) d y \\
= & \frac{1}{2}\left(D_{i}(2,1)+D_{i+1}(2,1)\right)\left(y_{i+1}-y_{i}\right) \frac{1}{2}\left(y_{i}+y_{i+1}\right) \\
& +\frac{1}{2}\left(D_{i}(2,1)+D_{i+1}(2,1)\right)\left(y_{i+1}-y_{i}\right) \frac{1}{3}\left(y_{i}^{2}+y_{i+1}^{2}+y_{i} y_{i+1}\right) \\
= & D_{i}(2,1) d \bar{\varepsilon}_{0}+D_{i}(2,2) d \bar{\kappa} \\
= & \frac{\partial M_{i}}{\partial \bar{\varepsilon}_{0}}+\frac{\partial M_{i}}{\partial \bar{\kappa}}
\end{aligned}
$$

where $y_{i}$ and $y_{i+1}$ are the position of each fiber depicted on Figure 3. The

sum of all contributions is included in the tangent stiffness matrix similar to (15), i.e.

$$
\mathbf{D}_{t}=\sum_{i=1}^{n}\left[\begin{array}{ll}
\frac{\partial N_{i}}{\partial \bar{\varepsilon}_{0}} & \frac{\partial N_{i}}{\partial \bar{\kappa}} \\
\frac{\partial M_{i}}{\partial \bar{\varepsilon}_{0}} & \frac{\partial M_{i}}{\partial \bar{\kappa}}
\end{array}\right]
$$


Appendix A.2. Implementation of foundation models

Based on the contribution to the variation in internal work $\delta \Omega$, from the beam and the two-parameter foundation, and the potential work of external forces $\delta W$ from point- and surface loads, the principle of virtual work for the system can be established

$$
\int_{V} \delta \boldsymbol{\varepsilon}^{T} \boldsymbol{\sigma}+\delta \boldsymbol{u}^{T} k \boldsymbol{u}+\delta \boldsymbol{\theta}^{T} \gamma \boldsymbol{\theta} d V=\int_{S} \delta \boldsymbol{u}^{T} \boldsymbol{f} d S+\sum_{i} \delta \boldsymbol{u}_{i}^{T} p_{i}
$$

where $V$ is the structural volume, $S$ is the surface area, $\delta \boldsymbol{u}$ and $\delta \boldsymbol{\theta}$ is the displacement and rotational variations, respectively, $\boldsymbol{f}$ is the surface traction vector, $p_{i}$ is a concentrated (nodal) load and $\delta \boldsymbol{u}_{i}$ is the associated (nodal) displacement variation. The contribution of the second parameter spring is here given on a general form considering $\gamma$ as a rotational stiffness. In case of a Winkler foundation type this latter term is omitted.

The beam-element and two-parameter foundation contribution to the internal nodal force, $\boldsymbol{q}$, is then given by the expression

$$
\boldsymbol{q}=\int_{0}^{L_{e}} \mathbf{B}^{T} \boldsymbol{\sigma} d x+\int_{0}^{L_{e}} \mathbf{N}^{T}\left[\begin{array}{cc}
k_{h} & 0 \\
0 & k_{v}
\end{array}\right] \mathbf{N} \boldsymbol{v}_{e} d x+\int_{0}^{L_{e}} \mathbf{G}^{T} \gamma \mathbf{I G} \boldsymbol{v}_{e} d x
$$

where $L_{e}$ is the length of the element, $\boldsymbol{v}_{e}$ is the global dof element displacements, $\mathbf{B}$ is the strain interpolation matrix and $\mathbf{N}$ is the displacement interpolation matrix.

The beam-element and two-parameter foundation contribution to the tangential stiffness matrix, $\mathbf{k}_{t}$, is given by the expression

$$
\mathbf{k}_{t}=\int_{0}^{L_{e}} \mathbf{B}^{T} \mathbf{D}_{t} \mathbf{B} d x+\int_{0}^{L_{e}} \mathbf{N}^{T}\left[\begin{array}{cc}
k_{h} & 0 \\
0 & k_{v}
\end{array}\right] \mathbf{N} d x+\int_{0}^{L_{e}} \mathbf{G}^{T} \gamma \mathbf{I G} d x
$$

The matrix $\mathbf{I}=\left[\begin{array}{ll}0 & 0 \\ 0 & 1\end{array}\right]$ is used to omit the axial terms in the second parameter interpolation matrix $\mathbf{G}$. Where $\mathbf{G}$ is given as $\mathbf{G}=\mathbf{N}^{\prime}$. 